\title{
The International Collaboration on Complementary Therapy Resources (ICCR): Working Together to Improve Online CAM Information
}

\author{
Karen Pilkington, $\mathrm{PhD}$, Are Gamst, MA, ${ }^{2}$ Irene Liu, MPH, ${ }^{3}$ Thomas Ostermann, $\mathrm{PhD},{ }^{4}$ \\ Dimity Pinto, $\mathrm{BS}^{5}$, and Janet Richardon, $\mathrm{PhD}^{6}$
}

\begin{abstract}
Background: Use of the Internet to find health information is increasing dramatically but the quality of information, particularly on complementary and alternative medicine (CAM) is variable. The International Collaboration on Complementary Therapy Resources (ICCR) involves collaboration between the national CAM information centers in Australia, Denmark, Norway, the United Kingdom, and the United States, and a CAM information service in Germany.

Objectives: The study objectives were to compare the services and areas of expertise offered by each of the national CAM information services, to explore common challenges encountered in practice, and to establish specific objectives for the collaboration.

Design: The workshop incorporated set templates for presentations, brainstorming, and analysis of notes to identify common themes.

Results: Differences and similarities between the various services were revealed. Common challenges were identified under the main themes of overall context, users and needs, content and processes. Based on these themes, it was possible to agree on a number of specific objectives.

Conclusions: The member organizations of the ICCR serve a range of different audiences and have varied remits to fulfill. For the international collaboration to be effective, it was necessary to identify common challenges and to agree on specific objectives and potential ways of working together. Progress to date is also discussed together with plans for the future.
\end{abstract}

\section{Introduction}

$\mathrm{C}$ URRENT ESTIMATES OF INTERNET USE suggest that $80 \%$ of American Internet users, or some 113 million adults, have searched for information on at least 1 of 17 health topics. ${ }^{1}$ Eight (8) million people use the Internet to search for health information on a typical day. However, there are several significant characteristics of Internet-based health information that need to be considered: There are no restrictions on who is able to present information or, for the majority of websites, on who is able to access the information. The format in which information is presented varies consider- ably, and there are no guarantees of the accuracy or currency of the content. Various studies have reported problems related to complementary and alternative medicine (CAM) information on the Internet. In each study, good-quality websites were found but information that was incomplete, inaccurate, out-of-date, or biased was also retrieved. ${ }^{2-11}$ A further consideration is that websites may be based anywhere in the world, so that the information may not be relevant to the reader and the health system to which he or she has access. Concerns have been expressed about the trust placed in the Internet by the general public: Three quarters of health information seekers do not consistently

\footnotetext{
${ }^{1}$ Department of Herbal Medicine and Nutritional Therapy, School of Life Sciences, University of Westminster, London, UK.

${ }^{2}$ National Information Center for Complementary and Alternative Medicine, Norway (NIFAB), NAFKAM, University of Tromsø, Tromsø, Norway.

${ }^{3}$ National Center for Complementary and Alternative Medicine, National Institutes of Health, U.S. Department of Health and Human Services, Bethesda, MD.

${ }^{4}$ Faculty of Health, Center for Integrative Medicine, University of Witten/Herdecke, Herdecke, Germany.

${ }^{5}$ National Institute of Complementary Medicine (NICM), University of Western Sydney, South Penrith, Australia.

${ }^{6}$ Faculty of Health, University of Plymouth, Plymouth, UK.
} 


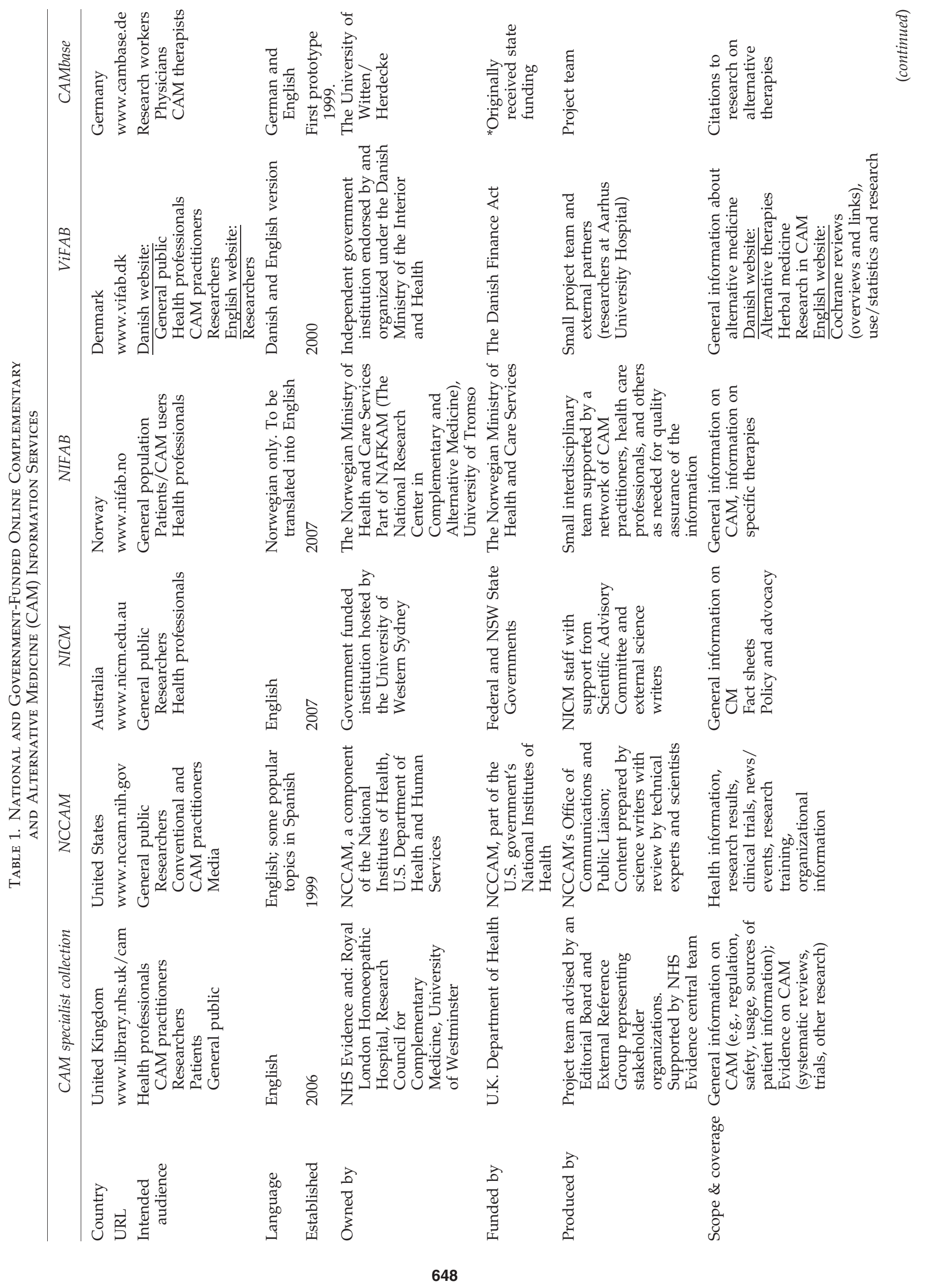




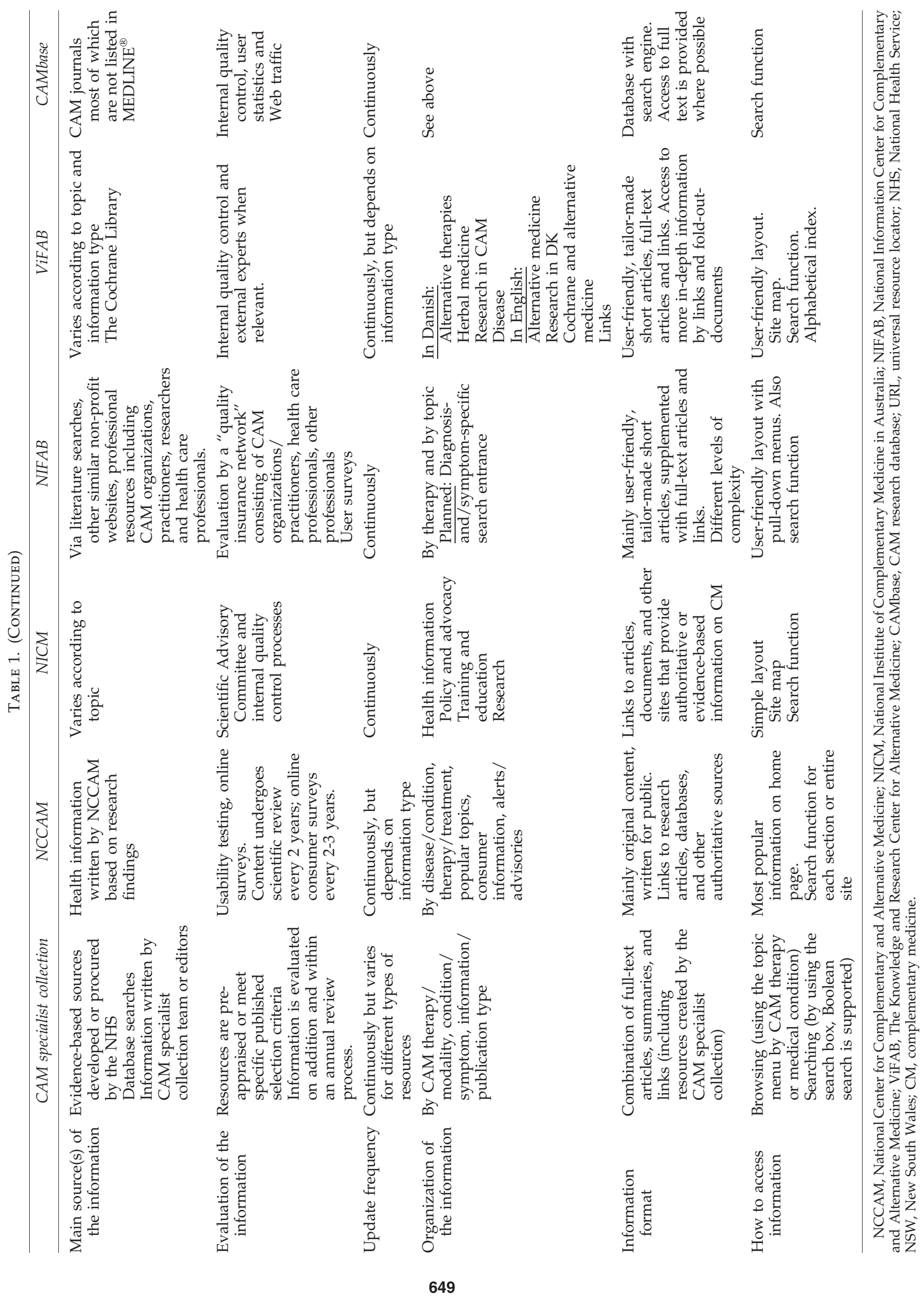


check the source and date of the health information they find online. ${ }^{1}$

Tools for evaluating websites are available, but there are some challenges with the use of these in practice. ${ }^{12-15}$ Even where good-quality research evidence is available, there may be considerable differences in how this information is presented on different websites. ${ }^{16}$

Consequently, the user searching for reliable information on CAM on the Internet is faced with a complex challenge related to assessing accuracy, relevance, and currency, together with recognizing commercial interests or biases. In several countries, this problem has been recognized and an attempt made to address the problem by means of the development of a national CAM online information service. National CAM websites, which are government funded, have been established in Australia, Denmark, Norway, the United Kingdom, and the United States. In Germany, government funding was provided to support the establishment of a CAM research database (CAMbase).

The first service to be established was the National Center for Complementary and Alternative Medicine (NCCAM at www.nccam.nih.gov) in the United States. This was launched in 1999 as part of the National Institutes of Health with the aim of funding research on CAM, training CAM researchers, and providing reliable information about CAM. The NCCAM website serves as the Center's primary medium for delivering CAM information to patients, the public, researchers, and health professionals. In 2001, NCCAM partnered with the National Library of Medicine to develop CAM on PubMed ${ }^{\circledR}$ - a subset of PubMed that contains citations to journal articles related to CAM. In the same year, the first prototype of the German CAMbase (www.CAMbase.de) database was developed by the University of Witten/ Herdecke supported with grants from the German Ministry of Research and Technology, the German Research Foundation, and private foundations. CAMbase aims to provide published information about complementary and alternative therapies while also offering an electronic document delivery service to researchers, physicians, and therapists. The focus is on CAM journals, most of which are not listed in MEDLINE. ${ }^{\circledR}$

A year later, the national Danish CAM website was launched. ViFAB (The Knowledge and Research Center for Alternative Medicine) is a nonprofit, independent institution endorsed by and organized under the Danish Ministry of the Interior and Health. The objectives of ViFAB are to promote knowledge of complementary and alternative therapies and herbal medicine and their effects, promote and develop research in this area, and encourage dialogue between health care providers, complementary and alternative therapists, researchers, and consumers. ViFAB's website, www .vifab.dk, serves as one of several means used to achieve these objectives.

In the United Kingdom, a Department of Health-funded project led to the development and launch of the Complementary and Alternative Medicine Evidence Online (CAMEOL) database (www.rccm.org.uk/cameol), comprising a series of systematic reviews of the evidence on specific complementary therapies in a range of conditions. Subsequently, the same organizations in collaboration with the Royal London Homoeopathic Hospital were awarded the contract to develop the National Library for Health CAM
Specialist Library. This is an electronic library collection of the best available current research evidence for CAM. In April 2009, the collection became part of NHS Evidence, an information service provided by the National Institute for Health and Clinical Excellence for all those working in health and social care in the United Kingdom. The collection is currently known as NHS Evidence-complementary and alternative medicine or the CAM specialist collection (www .library.nhs.uk/cam).

One of the more recently established services is NIFAB, the National Information Center for Complementary and Alternative Medicine, based in Norway (www.NIFAB.no). This is a national Web-based information center, the main aim of which is to support the Norwegian population in making safe and informed choices regarding CAM use. The most recently established service is NICM, the National Institute of Complementary Medicine in Australia (www.nicm .edu.au).

While each of the services has a specific task or area of activity officially assigned to meet based on national needs, there is obviously some duplication of effort involved. An initial discussion led to an informal collaboration between four national online CAM information providers and CAMbase being established in autumn 2007. The aim of this collaboration was to bring together providers of national, government-funded, Internet-based resources on CAM in order to facilitate the sharing and further development of these resources. This article focuses on the findings of a preliminary exercise to explore similarities, differences, and common challenges between the services.

\section{Objectives}

- To compare the services and areas of expertise offered by each of the national CAM information services

- To explore common challenges encountered in practice leading to the identification of areas for potential collaborative work

- To establish specific objectives for the collaboration

\section{Materials and Methods}

Prior to the initial meeting of representatives from the national CAM information centers, each service provided standard information on a pre-prepared template. In addition, each service was asked to identify specific challenges encountered in practice. Information was presented to the group, using the major headings Background, Methods, Results, Challenges. The group was divided into two and a workshop then took place, with each group exploring the common challenges. Brainstorming techniques were used and all points were noted. Two (2) members of the group analyzed the notes for common themes. Key points were tabulated and re-presented to the whole group. A guided discussion then took place on each of the themes to further understand different perspectives and to assess the potential for collaboration.

\section{Results}

The results are based on a summary of the presentations, group work, and subsequent discussions. 


\section{Key aspects of each of the information services}

Table 1 summarizes the main aspects of each website.

\section{Similarities and differences}

All of the organizations agreed that the overall aim of their websites is to make good-quality, reliable information more readily available. However, the discussion led to the identification of a number of similarities and differences between the services. These could be broadly represented under the following headings.

Audience. Virtually all information on each website is open access, but the primary intended audiences differed across the services. NCCAM, the longest established service, addresses information needs of the public, health professionals, and researchers as well as the media. The primary target for NIFAB and $\mathrm{ViFAB}$ is the general population, although the needs of other groups are also addressed. Conversely, CAMbase is intended for researchers, physicians, and therapists. In the United Kingdom, the CAM specialist collection has the NHS as its main audience but also aims to meet the needs of researchers and CAM practitioners. Other services in the United Kingdom such as NHS Direct and, more recently, NHS Choices provide an information service for patients and the general public, although not specifically on CAM. NICM's intended audience includes the general public, researchers, and health professionals.

Language. All websites include at least some information in English. Some information is presented in English on the NIFAB and ViFAB websites, but the primary languages for the services are Norwegian and Danish, respectively. Both NIFAB and ViFAB intend to translate further information into English in the future. CAMbase includes both English and German bibliographic references and an English version of its homepage. The CAM specialist collection and NCCAM present information in English, although the latter service does translate some consumer information into Spanish.

Stage of development. All the websites are at different stages of development, resulting in a variety of challenges. NCCAM and ViFAB were the first services to be established, whereas NICM is the most recent. The rate of development has varied because it is dependent on the extent of funding and consequently, staffing levels for each service. Because of this, the focus of the services differs; for example, the CAM specialist collection prioritizes provision of evidence in the form of citations of systematic reviews, while other services put greater emphasis on more general background or introductory information on specific therapies and approaches.

Health care and political context. The health care and political context within which each resource exists varies by country. The different environments impact on availability of therapies and traditional use of CAM. Prevalence of diseases varies and consequently, patterns of CAM use for specific diseases differ. The organization of health care within each country affects access to CAM, the extent of integration, and differences in legislation and regulation. Costs for particular therapies and treatments vary as do attitudes to and interest in CAM from the population at large and from the media and politicians.

\section{Challenges encountered}

During the group work, a number of common challenges were identified that could be organized into five main themes. These themes and the specific points were as detailed below.

\section{Overall context}

- Working within government-linked frameworks

- Multiple CAM organizations existing in each country

- Managing services within time and financial resources

The group agreed that it was important to provide a balanced and objective perspective. Because of the unique context and source of the funding, the websites are high profile and therefore are scrutinized closely by all the various stakeholder organizations. There is a need to be both evidence-based and experience-based and to incorporate opinions, experience, and expectations appropriately. In all countries, numerous CAM organizations exist, and thus stakeholders have wide-ranging perspectives and approaches. Among the government-funded services, there was a general recognition that resources are limited and each service must make the most effective use of funds.

\section{Users and needs}

- Gathering accurate information on and an understanding of usage and needs

- Meeting the needs of a range of different groups: patients, practitioners, health professionals, and researchers

- Dealing with queries related to individual patients/ consumers appropriately

- Keeping up to date with patients/consumers and with media interests

In most cases, each website is directed at a specific group or groups, but the nature of the Internet and the fact that the majority of content is open access means that the content can be accessed by a much wider range of people. The group discussed the difficulty in obtaining information about the users but acknowledged the importance of gaining a clear picture of the users through Web statistics and customer satisfaction surveys. A further consideration is that the development of the Internet and associated technology is rapid. CAM is also a constantly changing field as new approaches become available while media attention stimulates the demand for information.

\section{Content}

- Deciding on content/scope

- Addressing gaps in CAM information appropriately

- Producing background information on CAM therapies that appropriately represents different perspectives and practices

- Interpreting evidence appropriately (particularly addressing clinical relevance)

- Appropriately summarizing existing information (e.g., Cochrane reviews)

The scope of CAM varies considerably from one country to another, but in all cases is extremely broad and changes 
over time. However, as has been highlighted by many previous studies, there are many gaps in the information and evidence available, and the group agreed that meeting these information gaps was a particular challenge. In most cases, the organizations have produced information specifically for the website and are summarizing or interpreting existing information and evidence. Ensuring that this is done effectively is a significant challenge.

\section{Processes}

- Translation

- Searching for and identifying relevant information

- Managing technical aspects

All organizations are faced with the problem of language, in some cases from one language to another but also from "research language" to appropriate language for the general public or for clinicians who do not have an extensive knowledge of CAM. This is reflected in the challenge of using suitable vocabulary and indexing throughout the site. The lack of consistency of indexing CAM information in the general literature, for example, in medical databases, also causes problems in initially locating information because information is "hidden." The other challenge related to processes concerns the infrastructure within which each website functions which, in some cases, causes restrictions in functions. The services must also consider the rapid advances in technology and how to best utilize these as they become available.

Other challenges. The remaining challenges are associated with legal issues and potential liability, effective marketing, and dissemination. Each website faces the challenge of being found by potential users, keeping the website "fresh," and encouraging its use. The final challenge is that of evaluation, both of the organization's own website and of other websites to which it may link. The latter is particularly relevant as linking implies some degree of endorsement of other websites, whether or not this is the intention.

\section{Discussion and Conclusions}

Collectively, member organizations of the ICCR serve a wide range of audiences in their own and, potentially, in other countries. For international collaboration to be effective, it was necessary to identify common challenges and to agree on specific objectives. The recognition that national CAM information services are likely to face similar challenges stimulated a discussion on potential collaboration. Using a structured approach at the initial meeting proved an effective way to clarify the specific areas in which collaboration would be most valuable. It also enabled each of the information services to gain a more complete understanding of the specific areas of expertise on offer within the other services and of the differences between the services. Further discussions that have focused on addressing each of the challenges have resulted in a set of action points. These have included establishing a common website (www.iccrinfo.org), the aim of which is to encourage the sharing of knowledge and expertise between CAM information providers and those intending to develop similar services in the future. This site will include a "toolbox" of documents and advice on specific aspects such as how to set up user surveys or search for CAM evidence. The ICCR also acts as a forum within which each organization can request advice on specific topics and share experiences. Priority areas for each organization for the coming year are shared between members. In the case of several of the organizations having one or more priorities in common, they may then plan to work together on the specific aspect(s) identified.

In 2009, the ICCR was given the opportunity to hold its first workshop at an international CAM conference (North American Research Conference on Complementary and Alternative Medicine [NARCCIM], Minneapolis). The workshop addressed a number of issues particularly relevant to researchers in the CAM field including services offered on the national CAM websites, searching for CAM research, and presenting research to the public. The NICM in Australia (www.nicm.edu.au) has now joined the ICCR and the membership has been extended to encourage associate membership for non-national CAM information services (such as Pediatric Complementary and Alternative Medicine Research Network [PedCAM], www.pedcam.ca). By doing this, the aim is to further expand the effort to improve CAM information on a worldwide basis.

Aims for the future include investigating ways of reducing duplication of effort. This includes assessing the feasibility of sharing background information on specific therapies or producing information on priority areas collaboratively. Other aims are to continue to function as an informal forum for sharing experiences, support, and advice while continuing to explore the most effective ways of presenting information to health professionals, patients, and the general public.

In May 2011, NHS Evidence launched a new specialist evidence service in the United Kingdom to replace the specialist collections, including the CAM specialist collection. The new service will provide access to new content, as well as content previously available within the individual specialist collections and will be available at www.evidence .nhs.uk

\section{Acknowledgments}

The authors wish to thank the following individuals for their contribution to the development of the ICCR and/or to the preparation of this article: Alan Bensoussan (Australia), Anelia Boshnakova (UK), Mona Berg Hansen (Norway), Ola Lillenes (Norway), Helle Lonroth (Denmark), Terje Nilssen (Norway), Christa Raak (Germany), Anette Ranneries (Denmark), Chris Thomsen (USA), and Jannie Würtz (Denmark). The opinions expressed in this article are those of the authors and do not necessarily reflect those of the organizations.

\section{Disclosure Statement}

No competing financial interests exist.

\section{References}

1. Fox S. Online Health Search 2006. Pew Internet \& American Life Project, Oct 29, 2006. Online document at: www .pewInternet.org/Reports/2006/Online-Health-Search-2006 .aspx? $r=1$ Accessed January 12, 2010. 
2. Sagaram S, Walji M, Bernstam E. Evaluating the prevalence, content and readability of complementary and alternative medicine (CAM) web pages on the Internet. Proc AMIA Symp 2002;672-676.

3. Schmidt K, Ernst E. "Alternative" therapies for HIV/AIDS: How safe is Internet advice? A pilot study. Int J STD AIDS 2002;13:433-435.

4. Matthews SC, Camacho A, Mills PJ, Dimsdale JE. The Internet for medical information about cancer: Help or hindrance? Psychosomatics 2003;44:100-103.

5. Schmidt K, Ernst E. Complementary/alternative medicine for diabetes: How good is advice offered on websites? Diabet Med 2003;20:247-251.

6. Schmidt K, Ernst E. Assessing websites on complementary and alternative medicine for cancer. Ann Oncol 2004;15: 733-742.

7. Molassiotis A, Xu M. Quality and safety issues of web-based information about herbal medicines in the treatment of cancer. Complement Ther Med 2004;12:217-227.

8. Ernst E, Schmidt K. 'Alternative' cures for depression: How safe are web sites? Psychiatry Res 2004;129:297-301.

9. Gunasekera V, Ernst E, Ezra DG. Systematic Internet-based review of complementary and alternative medicine for glaucoma. Ophthalmology 2008;115:435-439.e2.

10. Whelan AM, Jurgens TM, Bowles SK, Doyle H. Efficacy of natural health products in treating osteoporosis: What is the quality of Internet patient advice? Ann Pharmacother 2009;43:899-907.

11. Brauer JA, El Sehamy A, Metz JM, Mao JJ. Complementary and alternative medicine and supportive care at leading cancer centers: A systematic analysis of websites. J Altern Complement Med 2010;16:183-186.
12. Walji M, Sagaram S, Sagaram D, et al. Efficacy of quality criteria to identify potentially harmful information: A crosssectional survey of complementary and alternative medicine web sites. J Med Internet Res 2004;6:e21.

13. Bernstam EV, Walji MF, Sagaram S, et al. Commonly cited website quality criteria are not effective at identifying inaccurate online information about breast cancer. Cancer 2008;112:1206-1213.

14. Bernstam EV, Sagaram S, Walji M, et al. Usability of quality measures for online health information: Can commonly used technical quality criteria be reliably assessed? Int J Med Inform 2005;74:675-683.

15. Breckons M, Jones R, Morris J, Richardson J. What do evaluation instruments tell us about the quality of complementary medicine information on the Internet? J Med Internet Res 2008;10:e3.

16. Hart A, Dey P. Echinacea for prevention of the common cold: An illustrative overview of how information from different systematic reviews is summarised on the Internet. Prev Med 2009;49:78-82.

Address correspondence to: Karen Pilkington, PhD Department of Herbal Medicine and Nutritional Therapy School of Life Sciences University of Westminster 115 New Cavendish Street London W1W 6UW

United Kingdom

E-mail: k.pilkington@westminster.ac.uk 
